\title{
LEITURA SPAD EM ABACAXIZEIRO IMPERIAL CULTIVADO EM DEFICIÊNCIA DE MACRONUTRIENTES E DE BORO ${ }^{1}$
}

\author{
MARIA JOSÉ MOTA RAMOS², PEDRO HENRIQUE MONNERAT ${ }^{3}$, \\ LEANDRO GLAYDSON DA ROCHA PINHO ${ }^{4}$
}

RESUMO- O equipamento Minolta SPAD-502 mede a intensidade da coloração verde das folhas e tem sido utilizado na quantificação de clorofilas, caracterizando-se pela rapidez, simplicidade e, principalmente, por possibilitar uma avaliação não destrutiva do tecido foliar. O objetivo deste trabalho foi calibrar a leitura SPAD, correlacionando-a com o diagnóstico das deficiências induzidas de macronutrientes e de boro associando às deficiências ao crescimento vegetativo do abacaxizeiro. O experimento constou de oito tratamentos: Completo, -N, -P, $-\mathrm{K},-\mathrm{Ca},-\mathrm{Mg}$, $-\mathrm{S}$ e -B, em blocos casualizados completos, com seis repetições. Foram avaliados o comprimento e a largura da folha " $\mathrm{D}$ " (marcada) e realizadas leituras com o medidor de clorofila SPAD 502. $\mathrm{O}$ uso do método de medida indireta da clorofila é adequado para a avaliação do estado nutricional de $\mathrm{N}$ e de crescimento vegetativo do abacaxizeiro 'Imperial'. O valor Spad e a concentração foliar de N no tratamento completo são, respectivamente: 75,7 e 14,8 $\mathrm{g} \mathrm{kg}^{-1}$, e no deficiente de $\mathrm{N}: 36,6$ e 9,7 $\mathrm{g} \mathrm{kg}^{-1}$. Com exceção das deficiências de $\mathrm{N}$ e $\mathrm{P}$, os demais tratamentos não afetaram a leitura SPAD.

Termos para indexação: Nitrogênio, clorofila, crescimento vegetativo.

\section{SPAD READING IN IMPERIAL PINEAPPLE UNDER MACRONUTRIENTES AND BORON DEFICIENCY}

\begin{abstract}
The equipment Minolta SPAD-502 measures the intensity of green color of leaves and has been used in the quantification of chlorophyll, characterized by speed, simplicity, and especially by allowing a non-destructive evaluation of the leaf tissue. The objective of this study was to calibrate the SPAD reading and its correlation with the diagnosis of induced deficiencies of macronutrients and boron deficiencies involving the vegetative growth of the pineapple. The experiment consisted of eight treatments: complete, $-\mathrm{N},-\mathrm{P},-\mathrm{K},-\mathrm{Ca},-\mathrm{Mg},-\mathrm{S}$ and $-\mathrm{B}$ in randomized complete block with six replicates. It was evaluated the length and the width of the sheet " $\mathrm{D}$ " (marked) and readings taken with the SPAD 502 chlorophyll meter. The use of the method of indirect measurement of chlorophyll is suitable for assessing the nutritional status of $\mathrm{N}$ and vegetative growth of 'Imperial' pineapple. The value Spad and concentration of leaf $\mathrm{N}$ in the complete treatment are respectively: 75.7 and $14.8 \mathrm{~g} \mathrm{~kg}^{-1}$ and deficient in $\mathrm{N}: 36.6$ and $9,7 \mathrm{~g} \mathrm{~kg}^{-1}$. With the exception of the deficiencies of $\mathrm{N}$ and $\mathrm{P}$, the other treatments did not affect SPAD readings.
\end{abstract}

Index terms: nitrogen, chlorophyll, vegetative growth.

\section{INTRODUÇÃO}

O equipamento Minolta SPAD-502, que mede a intensidade da coloração verde das folhas, tem sido utilizado na quantificação de clorofilas, caracterizando-se pela rapidez, simplicidade e, principalmente, por possibilitar uma avaliação não destrutiva do tecido foliar. Este equipamento tem sido utilizado com sucesso para diagnosticar o estado nutricional de diversas espécies frutíferas, como macieira (NEILSEN et al., 1995; PORRO et al., 2001a), videira (PORRO et al., 2001a e 2001b;
RUPP et al., 1999; RUPP; TRANKLE, 1995), pessegueiro (THOMIDIS; TSIPOURIDIS, 2005), cítrus (ARAÚJO et al., 2004).

Segundo Fontes et al. (1997), esse teor é estimado indiretamente pela intensidade da cor verde (unidades SPAD), medida em clorofilômetros, e o uso dessas medidas para determinar o teor de nitrogênio tem sido muito empregado nos últimos anos.

A atividade fotossintética, o conteúdo de proteínas solúveis de nitrogênio $(\mathrm{N})$, outros macronutrientes e micronutrientes existentes nas folhas são variáveis e podem ser correlacionadas com o

\footnotetext{
'(Trabalho 121-12). Recebido em: 23-03-2012. Aceito para publicação em: 007-01-2013.

${ }_{2}^{2}$ Pesquisadora da EMPAER/MT, Rua do Pintado S/N, Ponte Nova, Várzea Grande -MT. E-mail: majumota@ig.com.br ${ }^{3}$ Professor titular aposentado da UENF/CCTA. Email: phmonnerat@yahoo.com.br

${ }^{4}$ Professor do IFES. E-mail:leandro.pinho@ifes.edu.br.
} 
conteúdo de clorofila no tecido foliar (RAJCAN et al., 1999). Por isso, o medidor de clorofila Minolta SPAD-502 tem sido investigado como instrumento para o rápido diagnóstico do estado nutricional de diversas culturas em relação ao conteúdo de $\mathrm{N}$, agregando vantagens, como a simplicidade no uso, além de possibilitar uma avaliação não destrutiva do tecido foliar (ARGENTA et al., 2001).

$\mathrm{O}$ fornecimento de $\mathrm{N}$ influencia o valor da leitura SPAD. Os efeitos do N na leitura SPAD são influenciados pelo fósforo $(\mathrm{P})$. Além de $\mathrm{N}$, outros elementos, como o enxofre (S), ferro (Fe) manganês $(\mathrm{Mn})$, provocam clorose nas folhas, em caso de deficiência, o que evidencia sua importância na síntese de clorofila (MALAVOLTA et al., 1997).

O objetivo deste trabalho foi calibrar a leitura SPAD, correlacionando-a com o diagnóstico de deficiências induzidas de macronutrientes e de boro, obtido através de análise química foliar e visual apresentado por plantas de abacaxizeiro cv. Imperial, associando as deficiências ao crescimento vegetativo.

\section{MATERIAL E MÉTODOS}

O experimento foi implantado em casa de vegetação do Setor de Nutrição Mineral de Plantas da UENF, em Campos dos Goytacazes-RJ, no dia 19 de dezembro de 2003, utilizando-se de mudas do abacaxizeiro 'Imperial' produzidas a partir de cultura de tecidos na Campo Biotecnologia Vegetal Ltda., Unidade Biofábrica/EMBRAPA-CNPMF. O experimento constou de oito tratamentos: Completo, -N, -P, -K, -Ca, -Mg, -S e-B, em blocos casualizados completos, com seis repetições. A unidade experimental constou de um vaso plástico contendo $14 \mathrm{~kg}$ de areia de praia previamente purificada, contendo uma muda de abacaxizeiro. A purificação consistiu na embebição da areia com ácido clorídrico diluído em água, na proporção de 1:4, durante cerca de quatro horas, em recipiente plástico de 60 litros e posterior lavagem com água pura até que o $\mathrm{pH}$ se estabilizasse em valor próximo de 5 , quando, então, fez-se uma lavagem final com água desionizada. As mudas tinham tamanho médio de $6 \mathrm{~cm}$ e, após o plantio, receberam $500 \mathrm{~mL}$ de água desionizada a cada dois dias, durante 15 dias, quando novas raízes começaram a ser emitidas. Todos os vasos passaram, então, a receber, três vezes por semana, $500 \mathrm{~mL}$ da solução completa que apresentava a seguinte composição,

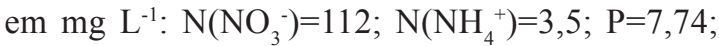
$\mathrm{K}=156,4 ; \mathrm{Ca}=80 ; \mathrm{Mg}=24,3 ; \mathrm{S}=32 ; 0 ; \mathrm{Cl}=1,77$; $\mathrm{Mn}=0,55 ; \mathrm{Zn}=0,13 ; \mathrm{Cu}=0,03 ; \mathrm{Mo}=0,05 ; \mathrm{B}=0,27$; $\mathrm{Fe}=2,23 \mathrm{com} \mathrm{pH}=5,5$. Após 50 dias de aplicação da solução completa ou 65 dias após o transplante, induziu-se a deficiência de boro, subtraindo-o dos vasos que receberam esse tratamento. Os demais tratamentos continuaram a receber a solução completa até 105 dias após o transplante, quando, então, reduziu-se a concentração dos macronutriente a $10 \%$ da solução completa. Aos 150 dias após o transplante, os tratamentos $-\mathrm{N},-\mathrm{P},-\mathrm{K},-\mathrm{Ca},-\mathrm{Mg}$ e $-\mathrm{S}$ tiveram seus respectivos nutrientes totalmente subtraídos da solução (Tabela 1). Aos 240 dias após o transplante, o tratamento $-\mathrm{N}$ voltou a receber solução nutritiva com $10 \%$ de $\mathrm{N}$ da solução completa, por 12 vezes, para evitar que as plantas ficassem impedidas de frutificar, futuramente. Os volumes de solução nutritiva aplicados variaram com o porte das plantas, sendo $500 \mathrm{~mL}$ até 150 dias após o plantio; $700 \mathrm{~mL}$, de 150 até 330 dias, e $1 \mathrm{~L}$ até a colheita, aplicados três vezes por semana.

Todas as plantas foram induzidas ao florescimento, oito meses após o plantio, quando as folhas " $D$ ", no tratamento completo, atingiram em média $63,4 \mathrm{~cm}$ de comprimento e com um número total de folhas de 55. Aplicou-se, na roseta foliar, por planta, $50 \mathrm{~mL}$ da solução de Etrel a $0,1 \%$, ureia a $2 \%$ e hidróxido de cálcio a $0,035 \%$ (VELOSO et al., 2001).

Foram avaliados o comprimento e a largura da folha "D" (marcada), e realizadas leituras com o medidor de clorofila SPAD 502 na face adaxial da folha, durante um mês, no período de $1^{\circ}$-09-2004 a 29-092004. As leituras com o SPAD foram realizadas em 10 pontos diferentes da folha, no final da tarde, logo após as avaliações de crescimento. Avaliaram-se também, aos nove meses após o plantio, na folha "D" inteira de cada planta, os teores de N, P, K, Ca, $\mathrm{Mg}$, S e B, conforme metodologia de Malavolta et al. (1997), modificada por Monnerat S/D.

Os dados foram submetidos à análise de variância, com arranjo fatorial $8 \times 4$ (oito tratamentos $\mathrm{x}$ quatro épocas de coleta). As médias dos tratamentos sob deficiência foram comparadas à do controle, pelo teste de Dunnet a 5\%. A análise de correlação foi realizada na época 4 (29-09-04), entre as avaliações de crescimento e teor de nutrientes, a 5\% de significância.

\section{RESULTADOS E DISCUSSÃO}

Observa-se que, na deficiência de $\mathrm{N}$, o valor Spad e o comprimento da folha ' $\mathrm{D}$ ' foram menores do que no tratamento completo e que a deficiência de P também reduziu o valor SPAD. Os demais nutrientes não afetaram significativamente a leitura SPAD (Tabela 2), embora as plantas apresentassem sintomas de deficiência (RAMOS et al., 2009). Cada nutriente deficiente reduziu seu próprio teor foliar, 
aos nove meses após o plantio. Ramos et al. (2009) observaram que, nas plantas submetidas à deficiência de N, no sétimo mês após o plantio do abacaxizeiro cv. Imperial, o ápice das folhas mais velhas começou a secar e, aos nove, este sintoma intensificou-se; além disto, as folhas eram mais amareladas.

Houve correlação linear e positiva da leitura SPAD com a largura da folha $\mathrm{D}$ e com a deficiência de $\mathrm{N}$, indicando que o índice SPAD pode ser usado na estimativa de crescimento vegetativo do abacaxizeiro e do status nutricional do N (Tabela 3). Prado e Valle (2008) consideraram o uso do método de medida indireta da clorofila adequado para a avaliação do estado nutricional de $\mathrm{N}$ no porta-enxerto de limoeiro-cravo e também com a produção de matéria seca. Decarlos Neto et al. (2002) relataram a ocorrência de correlação positiva entre a aplicação de $\mathrm{N}$ e a leitura SPAD, em plantas de porta-enxertos de citros.

Em caramboleira, plantas não adubadas apresentaram a menor leitura SPAD em relação àquelas que receberam o fertilizante nitrogenado (LEAL et al., 2007).

A relação entre leitura SPAD e teor de $\mathrm{N}$ é atribuída, principalmente, ao fato de $50 \%$ a $70 \%$ do $\mathrm{N}$ total das folhas serem integrantes de compostos associados aos cloroplastos e ao conteúdo de clorofila das folhas (CHAPMAN ; BARRETO, 1997).

Segundo Argenta et al. (2001), a leitura realizada com clorofilômetro nas folhas do milho estima com boa precisão o teor de clorofila, sendo eficiente parâmetro para separar plantas com deficiência e com nível adequado de nitrogênio.

O nitrogênio possui função estrutural na planta, sendo fundamental para o crescimento vegetativo, estimulando o desenvolvimento de gemas floríferas e frutíferas, além de aumentar a produção de fotoassimilados e, consequentemente, a produção (BHELLA e WILCOX, 1986). É também necessário à síntese da clorofila e, por ser parte integrante dessa molécula, está envolvido no processo de fotossíntese. Sendo assim, na falta de nitrogênio e, consequentemente, de clorofila, a planta não utiliza a luz do Sol como fonte de energia para realizar algumas funções essenciais, como a absorção de nutrientes e a produção de carboidratos para o seu desenvolvimento, florescimento e produção de frutos.

Shaahan et al. (1999) observaram que o índice relativo ao teor de clorofila, geralmente, correlaciona-se bem com o teor de $\mathrm{N}$ nas folhas, podendo indicar deficiência de N nas plantas (WOOD et al., 1993).

TABELA 1 - Composição química da solução nutritiva completa e com omissão dos macronutrientes e do boro $\left(\mathrm{mL} \mathrm{L}^{-1}\right)$.

\begin{tabular}{|c|c|c|c|c|c|c|c|c|}
\hline Soluções estoques & Completa & $-\mathbf{N}$ & $-\mathbf{P}$ & $-\mathbf{K}$ & $-\mathrm{Ca}$ & $-\mathrm{Mg}$ & $-\mathrm{S}$ & $-B$ \\
\hline $\mathrm{Ca}\left(\mathrm{NO}_{3}\right)_{2-} 4 \mathrm{H}_{2} \mathrm{O} 2 \mathrm{~mol} \mathrm{~L}^{-1}$ & 1 & & 1 & 1 & & 1 & 1 & 1 \\
\hline $\mathrm{KNO}_{3} 2 \mathrm{~mol} \mathrm{~L}^{-1}$ & 2 & & 2 & & 2 & 2 & 2 & 2 \\
\hline $\mathrm{MgSO}_{4} 7 \mathrm{H}_{2} \mathrm{O} 1 \mathrm{~mol} \mathrm{~L}^{-1}$ & 1 & 1 & 1 & 1 & 1 & & & 1 \\
\hline$\left(\mathrm{NH}_{4}\right) \mathrm{H}_{2} \mathrm{PO}_{4} 1 \mathrm{~mol} \mathrm{~L}^{-1}$ & 0,25 & & & 0,25 & 0,25 & 0,25 & 0,25 & 0,25 \\
\hline $\mathrm{H}_{3} \mathrm{BO}_{3}$ & 1 & 1 & 1 & 1 & 1 & 1 & 1 & \\
\hline Fe-EDTA & 1 & 1 & 1 & 1 & 1 & 1 & 1 & 1 \\
\hline Micro -B -Fe & 1 & 1 & 1 & 1 & 1 & 1 & 1 & 1 \\
\hline $\mathrm{CaCl}_{2} 2 \mathrm{~mol} \mathrm{~L}^{-1}$ & & 1 & & & & & & \\
\hline $\mathrm{NaNO}_{3} 2 \mathrm{~mol} \mathrm{~L}^{-1}$ & & & & 2 & 2 & & & \\
\hline $\mathrm{KCL} 1 \mathrm{~mol} \mathrm{~L}^{-1}$ & & 3,75 & & & & & & \\
\hline $\mathrm{Na}_{2} \mathrm{SO}_{4} 1 \mathrm{~mol} \mathrm{~L}^{-1}$ & & & & & & 1 & & \\
\hline $\mathrm{KH}_{2} \mathrm{PO}_{4} 1 \mathrm{~mol} \mathrm{~L}^{-1}$ & & 0,25 & & & & & & \\
\hline $\mathrm{MgCl}_{2} 1 \mathrm{~mol} \mathrm{~L}^{-1}$ & & & & & & & 1 & \\
\hline $\mathrm{NH}_{4} \mathrm{Cl} 1 \mathrm{~mol} \mathrm{~L}^{-1}$ & & & 0,25 & & & & & \\
\hline
\end{tabular}

*Para o preparo de $1 \mathrm{~L}$ de solução de micronutrientes foram utilizados os seguintes reagentes analíticos em g: $\mathrm{KCl}: 3,7467 ; \mathrm{MnSO} 4$ $2 \mathrm{H}_{2} \mathrm{O}: 0,8451 ; \mathrm{ZnSO}_{4} 7 \mathrm{H}_{2} \mathrm{O}: 0,5780 ; \mathrm{CuSO}_{4} 5 \mathrm{H}_{2} \mathrm{O}: 0,1267$ e $\left(\mathrm{NH}_{4}\right)_{6} \mathrm{Mo}_{7} \mathrm{O}_{24} 4 \mathrm{H}_{2} \mathrm{O}: 0,088$. 
TABELA 2-Concentração foliar de macronutrientes $\left(\mathrm{g} \mathrm{kg}^{-1}\right)$ e boro $\left(\mathrm{mg} \mathrm{kg}^{-1}\right)$, leitura SPAD, comprimento e largura da folha D no abacaxizeiro' Imperial', nos tratamentos completo e com indução de deficiência, aos 9 meses após o plantio.

\begin{tabular}{|c|c|c|c|c|c|c|}
\hline Tratamentos & Nutriente & Concentração & Leitura Spad & $\begin{array}{c}\text { Comprimento } \\
(\mathrm{cm})\end{array}$ & Largura & CV $(\%)$ \\
\hline Completo & & 14,8 & 75,7 & 66,2 & $4,68 \mathrm{~ns}$ & 8,13 \\
\hline$-\mathrm{N}$ & $\mathrm{N}$ & $9,7-$ & $36,6-$ & $54,7-$ & $3,60 \mathrm{~ns}$ & \\
\hline Completo & & 1,23 & & & & 11,3 \\
\hline$-\mathrm{P}$ & $\mathrm{P}$ & $0,67-$ & $65,6-$ & $64,9 \mathrm{~ns}$ & $6,87 \mathrm{~ns}$ & \\
\hline Completo & & 20,0 & & & & 9,05 \\
\hline$-K$ & K & $11,6-$ & $77,8 \mathrm{~ns}$ & $59,3 \mathrm{~ns}$ & $4,36 \mathrm{~ns}$ & \\
\hline Completo & & 4,37 & & & & 15,6 \\
\hline$-\mathrm{Ca}$ & $\mathrm{Ca}$ & $0,91-$ & $74,0 \mathrm{~ns}$ & $59,3 \mathrm{~ns}$ & $4,72 \mathrm{~ns}$ & \\
\hline Completo & & 2,26 & & & & 12,0 \\
\hline$-\mathrm{Mg}$ & $\mathrm{Mg}$ & $0,73-$ & $74,2 \mathrm{~ns}$ & $61,3 \mathrm{~ns}$ & $4,66 \mathrm{~ns}$ & \\
\hline Completo & & 1,45 & & & & 16,2 \\
\hline$-S$ & $\mathrm{~S}$ & $0,45-$ & $70,4 \mathrm{~ns}$ & $67,4 \mathrm{~ns}$ & $4,82 \mathrm{~ns}$ & \\
\hline Completo & B & 18,4 & & & & 11,5 \\
\hline$-B$ & & $5,8-$ & $74,9 \mathrm{~ns}$ & $62,0 \mathrm{~ns}$ & $4,90 \mathrm{~ns}$ & \\
\hline CV (\%) & & & 8,11 & 9,87 & 8,42 & \\
\hline
\end{tabular}

Em cada coluna, médias seguidas por + , - ou ns são maiores, menores ou não diferem do tratamento completo, respectivamente, pelo teste de Dunnett, a $5 \%$.

TABELA 3- Correlação entre SPAD, comprimento (C) e largura (L) da folha ' $D$ ' e teor dos macronutrientes e de boro na cv. Imperial em deficiência nutricional.

\begin{tabular}{|c|c|c|c|c|c|c|c|c|c|c|}
\hline & $\mathrm{C}$ & $\mathrm{L}$ & SPAD & $\mathrm{N}$ & $\mathrm{P}$ & $\mathrm{K}$ & $\mathrm{Ca}$ & $\mathrm{Mg}$ & $\bar{S}$ & $\mathrm{~B}$ \\
\hline $\mathrm{C}$ & & 0,691 & 0,441 & 0,301 & $-0,325$ & $-0,051$ & 0,196 & $-0,335$ & $-0,402$ & $-0,057$ \\
\hline $\mathrm{L}$ & & & $0,753 *$ & 0,633 & $-0,496$ & $-0,245$ &,- 072 & $-0,148$ & $-0,126$ & $-0,576$ \\
\hline SPAD & & & 1,00 & 0,916 ** & $-0,546$ & $-0,656$ & $-0,003$ & $-0,132$ & 0,2967 & $-0,394$ \\
\hline $\mathrm{N}$ & & & & & $-0,344$ & $-0,794 * *$ & 0,1629 & $-0,065$ & 0,266 & $-0,273$ \\
\hline $\mathrm{P}$ & & & & & & 0,367 & 0,356 & 0,089 & 0,132 & 0,596 \\
\hline K & & & & & & & $-0,154$ & $-0,292$ & $-0,209$ & 0,238 \\
\hline $\mathrm{Ca}$ & & & & & & & & $-0,526$ & 0,342 & 0,092 \\
\hline $\mathrm{Mg}$ & & & & & & & & & $-0,231$ & $-0,038$ \\
\hline $\mathrm{S}$ & & & & & & & & & & $-0,202$ \\
\hline
\end{tabular}

*: significativo a 1 e $5 \%$ de probabilidade pelo teste $\mathrm{t}$.

\section{CONCLUSÕES}

1- O uso do método de medida indireta da clorofila é adequado para a avaliação do estado nutricional de $\mathrm{N}, \mathrm{P}$ e de crescimento vegetativo do abacaxizeiro 'Imperial'.

2- O valor Spad e a concentração foliar de N, no tratamento completo, são, respectivamente: 75,7 e $14,8 \mathrm{~g} \mathrm{~kg}^{-1}$, e no deficiente de $\mathrm{N}: 36,6$ e $9,7 \mathrm{~g} \mathrm{~kg}^{-1}$.

3- $O$ valor Spad e a concentração foliar de $P$, no tratamento completo, são, respectivamente: 75,7 e $1,23 \mathrm{~g} \mathrm{~kg}^{-1}$, e no deficiente de P: 65,3 e $0,67 \mathrm{~g} \mathrm{~kg}^{-1}$.

4- Os teores foliares das plantas normais e deficientes, no início da formação dos frutos, foram, respectivamente: $\mathrm{N}=14,8$ e 9,$7 ; \mathrm{P}=1,23$ e 0,$67 ; \mathrm{K}=$ 20,0 e 11,$6 ; \mathrm{Ca}: 4,37$ e 0,$91 ; \mathrm{Mg}=2,26$ e 0,$73 ; \mathrm{S}=$ 1,45 e $0,45 \mathrm{~g} \mathrm{~kg}^{-1}$ e $\mathrm{B}=18,4$ e $5,8 \mathrm{mg} \mathrm{kg}^{-1}$.

\section{REFERÊNCIAS}

ARAÚJO, R.A. de; SIQUEIRA, D.L. de; MARTINEZ, C.A.; FERNANDES, A.R. Características biométricas, índice SPAD-502 e emissão da fluorescência em porta-enxertos de citros. Revista Ceres, Viçosa, MG, v.51, n.294, p.189-199, 2004.

ARGENTA, G.; SILVA, P. R. F. da; BARTOLINI, C. G.; FORSTHOFER, E. L.; STRIEDER, M. L. Relação da leitura do clorofilômetro com os teores de clorofila extraível e nitrogênio na folha de milho.

Revista Brasileira de Fisiologia Vegetal, Lavras, v. 13, n. 2, p. 158-167, 2001. 
BHELLA, M.; WILCOX, G.E. Yield and composition of muskmelon as influenced by preplant and trickle applied nitrogen. Hort Science, Alexandria, v.21, p.86-88, 1986.

CHAPMAN, S. C.; BARRETO, H. J. Using a chlorophyll meter to estimate specific leaf nitrogen of tropical maize during vegetative growth. Agronomy Journal, Madison, v. 89, n.1, p. 557-562, 1997.

DECARLOS NETO, A. et al. Crescimento de porta-enxertos de citros em tubetes influenciados por doses de N. Revista Brasileira de Fruticultura, Jaboticabal, v. 24, n. 1, p. 199-203, 2002.

FONTES, P.C.R.; PEREIRA, P.R.G.; CONDE, R.M. Critical chlorophyll, total N, and NO3--N in leaves associated to maximum lettuce yield. Journal of Plant Nutrition, New York, v.20, p.1.061-1.068, 1997.

LEAL, R.M.; NATALE, W.; , R. de M.; ZACCARO, R.P. Adubação nitrogenada na implantação e na formação de pomares de caramboleira. Pesquisa Agropecuária Brasileira, Brasília, v.42, n.8, p.11111119, 2007.

MALAVOLTA, E.; VITTI, G.C.; OLIVEIRA, S.A. Avaliação do estado nutricional das plantas. 2.ed. Piracicaba: Potafós, 1997. 319p.

NEILSEN, D.; HOGUE, E.J.; NEILSEN, G.H.; PARCHOMCHUK, P. Using SPAD-502 values to assess the nitrogen status of apple trees. HortScience, Alexandria, v.30, n.3 p.508-512, 1995.

PORRO, D.; BERTAMINI, M.; DORIGATTI, C.; STEFANINI, M.; CESCHINI, A. Lo SPAD nella diagnosi dello stato nutrizionale della vite. Informatore Agrario, Verona, v.57, n.26, p.49-55, 2001 b.

PORRO, D.; DORIGATTI, C.; STEFANINI, M.; CESCHINI, A. Use of SPAD meter in diagnosis of nutritional status in apple and grapevine. Acta Horticulturae, The Hague, n.564, p.243-252, 2001a.

PRADO, R. de M.; VALE, D. W. do. Nitrogênio, fósforo e potássio na leitura spad em porta-enxerto de limoeiro-cravo. Pesquisa Agropecuária Tropical, Goiânia, v. 38, n. 4, p. 227-232, out./dez. 2008.
RAJCAN, I.; DWYER, L.; TOLLENAAR, M. Note on relationship between leaf soluble carbohydrate and chlorophyll concentrations in maize during leaf senescence. Field Crops Research, Madison, v. 63, p. 13-17, 1999.

RAMOS, M.J.M.; MONNERAT, P.H.; CARVALHO, A.J.C. de; PINTO, J.L. A.; SILVA, J.A da.Sintomas visuais de macronutrientes e de boro em abacaxizeiro 'Imperial'. Revista Brasileira de Fruticultura, Jaboticabal, v.31, n.1, p.252-256, 2009.

RUPP, D.; TRANKLE, L. A non-destructive measurement method for chlorophyll in grapevines. Mitteilungen Klosterneuburg, Rebe und Wein, Obstbau und Fruechteverwertung, v.45, n.5/6, p.139-142, 1995.

RUPP, D.; TRANKLE, L.; FOX, R. Non-destructive measurement of chlorophyll in grapes - evaluation of varietal influences and effects of sampling methods. Mitteilungen Klosterneuburg, Rebe und Wein, Obstbau und Fruechteverwertung, v.49, n.3, p.86-92, 1999.

SHAAHAN, M.M.; EL-SAYED, A.A.; ABOU ELNOUR, E.A.A. Predicting nitrogen, magnesium and iron nutritional status of perennial crops using a portable chlorophyll meter. Scientific Horticulture, Kent, v. 82, p. 339-348, 1999.

THOMIDIS, T.; TSIPOURIDIS, C. Influence of rootstocks, $\mathrm{pH}$, iron supply (in nutrient solutions) and Agrobacterium radiobacter on chlorophyll and iron concentration in leaves of a peach variety. Journal of Plant Nutrition, Abingdon, v.28, n.10, p.1.8331.842, 2005.

VELOSO, C.A.C.; OEIRAS, A.H.L.;CARVALHO, E.J.M.; SOUZA, F.R.S. Resposta do abacaxizeiro à adição de nitrogênio, potássio e calcário em Latossolo Amarelo do nordeste paraense. Revista Brasileira de Fruticultura, Jaboticabal, v. 23, p. 396-402, 2001.

WOOD, C.W.; REEVES, D.W.; HIMELRICK, D.G. Relationship between chlorophyll meter readings and leaf chlorophyll concentration, $\mathrm{N}$ status and crop yield: a review. Proceedings Agronomy Society of New Zealand, Ashburton, v. 23, p. 1-9, 1993. 